\title{
Development of a simple multidisciplinary arthroplasty wound-assessment instrument: the SMArt Wound Tool
}

\author{
Sahil Singh Kooner, MD \\ Brendan Sheehan, MD \\ Joseph Keith Kendal, MD \\ Herman Johal, MD
}

Accepted Dec. 6, 2017; Early-released

Aug. 1, 2018

\section{Correspondence to:}

\section{S. Kooner}

Department of Orthopedics

University of Calgary

1122 3rd St SE

Calgary $A B$ T2G $1 \mathrm{H} 7$

sahilkooner@gmail.com

DOI: $10.1503 /$ cjs. 015017
Background: There are currently no validated instruments in the orthopedic literature for assessing the healing of acute surgical wounds. The creation of a simple wound-assessment tool would provide a standardized method of reporting wound outcomes. The objective of this study was to systematically develop a wound-assessment tool that can be used to assess the early healing of arthroplasty incisions.

Methods: The databases MEDLINE, Embase, Cochrane Central Register of Controlled Trials, Cochrane reviews and CINAHL were searched. Articles that described objective assessment of acute incisional wounds were included. Items for the woundassessment tool were then extracted from eligible studies based on the frequency of reporting. A multidisciplinary panel of wound experts compiled the items into an initial tool to assess key domains of wound healing. The items were reduced through several iterations of panel discussion.

Results: Our search strategy yielded 3743 results, which were screened by title and abstract. Thirty-four studies were included in the systematic review for the development of the wound-assessment tool, and 10 domains were extracted based on frequency of reporting. After item reduction, the final version of the wound-assessment tool, the SMArt Wound Tool, contained 3 major domains: blistering, peri-incisional skin colour and exudate type.

Conclusion: There is currently a need for a standardized tool to assess the healing of orthopedic surgical incisions. The SMArt Wound Tool provides a simple, objective method of assessing arthroplasty incisions for the presence of early complications.

Contexte : Il n'existe actuellement aucun instrument validé dans la littérature orthopédique pour évaluer la cicatrisation des plaies chirurgicales récentes. La création d'un outil d'évaluation simple des plaies offrirait une méthode standard pour suivre leur évolution. L'objectif de cette étude était de concevoir un outil d'évaluation systématique des plaies pouvant être utilisé pour vérifier la bonne cicatrisation des incisions d'arthroplastie.

Méthodes : Les bases de données MEDLINE, Embase, CINAHL, le registre central Cochrane des essais contrôlés et la base de données Cochrane des revues systématiques ont été interrogés. Les articles qui décrivaient une évaluation objective des plaies d'incision récentes ont été inclus. Les paramètres d'évaluation des plaies ont ensuite été extraits à partir des études retenues en fonction de la fréquence à laquelle ils étaient rapportés. Un comité multidisciplinaire d'experts des plaies a compilé les paramètres pour créer un outil initial d'évaluation des domaines clés de la cicatrisation des plaies. Des discussions successives du comité ont ensuite permis de réduire le nombre de paramètres d'évaluation.

Résultats : Notre stratégie d'interrogation a généré 3743 résultats qui ont été triés par titre et résumé. Trente-quatre études ont été retenues pour la revue systématique en vue de la création de l'outil d'évaluation des plaies et 10 domaines ont été extraits en fonction de la fréquence à laquelle ils étaient rapportés. Après la réduction du nombre de paramètres, la version finale de l'outil d'évaluation des plaies, appelé SMArt Wound Tool, comprenait 3 domaines principaux : formation de vésicules, couleur de la peau au pourtour de l'incision et type d'exsudat.

Conclusion : On déplore actuellement l'absence d'outils standards pour évaluer la cicatrisation des plaies en chirurgie orthopédique. L'outil SMArt offre une méthode simple et objective d'évaluation des incisions d'arthroplastie pour déceler rapidement la moindre complication, le cas échéant. 


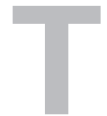
otal hip and knee arthroplasty are common operations, with about 2 million procedures performed annually worldwide. ${ }^{1}$ This number is expected to increase substantially in the future owing to an aging population that is living increasingly active and longer lives. As a consequence, the burden of total joint arthroplasty (TJA) complications is expected to increase as well. ${ }^{2}$ Revision surgery is arguably one the most serious complications associated with TJA, and reasons for revision can be diverse and varied. Among these causes, prosthetic joint infection is one of the most morbid complications. ${ }^{3}$ Total knee infection rates range from $1.1 \%$ to $2.2 \%$, and such infections account for just over $25 \%$ of total knee arthroplasty revision procedures. ${ }^{4}$ Despite the best efforts of health care professionals, TJA infection rates have remained remarkably constant. ${ }^{4}$

Because of these issues, nearly all aspects of perioperative and postoperative care have been standardized to reduce the risk of preventable postoperative complications. For this reason, clinical pathways and best-practice guidelines have become increasingly common in surgical care, emphasizing appropriate antibiotics preoperatively, sterile technique and ongoing wound care. ${ }^{5}$ Given the impact of wound healing on patient outcome, accurate and standardized assessment of acute incisional wounds is not only important but also necessary as a screening tool to identify those at risk for early postoperative complications. ${ }^{6}$

To our knowledge, there are no validated instruments in the orthopedic literature that assess healing of acute incisional wounds by primary intention. Given the burden of infection and importance of wound healing in TJA, it is surprising that such a tool has not yet been developed. Institutional wound-assessment tools are rarely evidence based, relying more on expert opinion and anecdotal evidence. Furthermore, different studies and institutions often use completely different wound-assessment tools, which makes comparison difficult. Wound assessment can be subjective without a systematic approach, which leads to ineffective communication about wound care in a multidisciplinary setting. ${ }^{7}$ Even seemingly objective measurements, such as the size of a wound, can vary widely between health care professionals without a means for standardization. ${ }^{8}$ Inconsistent terminology, poor documentation and lack of uniformity between different health care professionals can lead to adverse outcomes for patients and come at a great cost to the health care system. ${ }^{\text {? }}$

The creation of a simple multidisciplinary woundassessment tool would allow for a standardized method of observing and reporting wound outcomes, potentially reducing medical errors and improving patient care. As such, the objective of this study was to systematically develop and validate a wound-assessment tool that can be used to assess the early healing of hip and knee arthroplasty incisions. We hope that such a tool can be easily applied by various members of the health care team, con- tain only the most pertinent information, and reliably and accurately evaluate wound healing in the acute postoperative period.

\section{Methods}

A systematic literature search was performed by 3 reviewers (B.S, H.J, J.K.K.) in February 2013 and updated in August 2017. The databases MEDLINE, Embase, Cochrane Central Register of Controlled Trials, Cochrane reviews and CINAHL were searched by means of enginespecific strategies unique to each database to maximize sensitivity (Appendix 1, available at canjsurg.ca/015017-a1). The reviewers explored references of each study and related citations on each search engine for any further eligible studies. All search results were then compiled in an online database, and duplicates were deleted. Studies were then scanned based on title and abstract for eligibility. For any title selected by the reviewers, the abstract was then reviewed. All abstracts selected by the reviewers were then subjected to a full manuscript review. Primary articles that met all inclusion and exclusion criteria were included in the systematic review. Disagreements were resolved by consensus via discussion among the reviewers.

Eligibility criteria for studies included case series, cohort studies, clinical trials or review articles that described the objective assessment of acute incisional wounds. Studies were excluded if only chronic or nonsurgical wounds were assessed, nonobjective assessment was described, the method of assessment was not described, only nonorthopedic wounds were assessed or articles were in a foreign language with no English-language translation available. If an article included reports on chronic incisions but wound-assessment criteria could be extracted, it was also included.

The included articles were evaluated for level of evidence and method of objective wound assessment. The methods were evaluated for standardization, type of wound assessed, inclusion of grading scale, validation and reproducibility.

We extracted items for the wound-assessment tool from eligible studies identified in the systematic review based on frequency of reporting. This list was then presented in its entirety to a multidisciplinary panel of wound-assessment experts consisting of wound care nurses, nurse practitioners, orthopedic surgeons and plastic surgeons. The panel compiled the items into an initial tool to assess key domains of each wound and developed appropriate rating scales. The items were reduced through several iterations of panel discussion and piloting of the tool among patients who had undergone hip or knee arthroplasty at their regularly scheduled 2 -week postoperative assessment. Items were evaluated for clinical practicality, efficiency of use, redundancy and overall applicability. Based on these qualities, the pilot form was then refined into a final woundassessment tool. 


\section{Results}

The search strategy yielded 4349 results (Fig. 1). Once duplicates were removed, the reviewers screened 3743 titles and 151 abstracts. Subsequently, 61 articles were selected for full-text review, of which 41 were excluded based on the inclusion and exclusion criteria. An additional 14 full texts were identified through references and were added for analysis. In total, 34 studies were included in the systematic review for development of the wound-assessment tool.

Studies included for analysis included randomized controlled trials, systematic reviews, prospective clinical trials, retrospective cohorts and narrative reviews. Most of the studies assessed nonorthopedic surgical incisions, and 11 studies included patients who underwent TJA. Nonetheless, only 5 studies described validated woundassessment methods: 2 were in the form of a visual ana- logue scale to assess the appearance of scars after acute surgical incisions, 2 described using the updated Centers for Disease Control and Prevention criteria for surgical site infection, and 1 described using an adapted ulcer grading scale for chronic wounds. In most studies, nonvalidated wound-assessment methods were used that were study or institution specific. In addition, wound-assessment terminology varied widely between studies, and exact definitions of wound-specific terminology were often not discussed. Owing to this ambiguity, there was an element of subjectivity required to group similar wound-assessment terms together for the purpose of extraction and frequency reporting.

From the studies analyzed, we extracted 10 criteria based on frequency of reporting. These 10 items included global wound assessment, skin colour, exudate type, exudate amount, odour, skin edge health, wound apposition, drainage, blisters and cosmetic appearance at

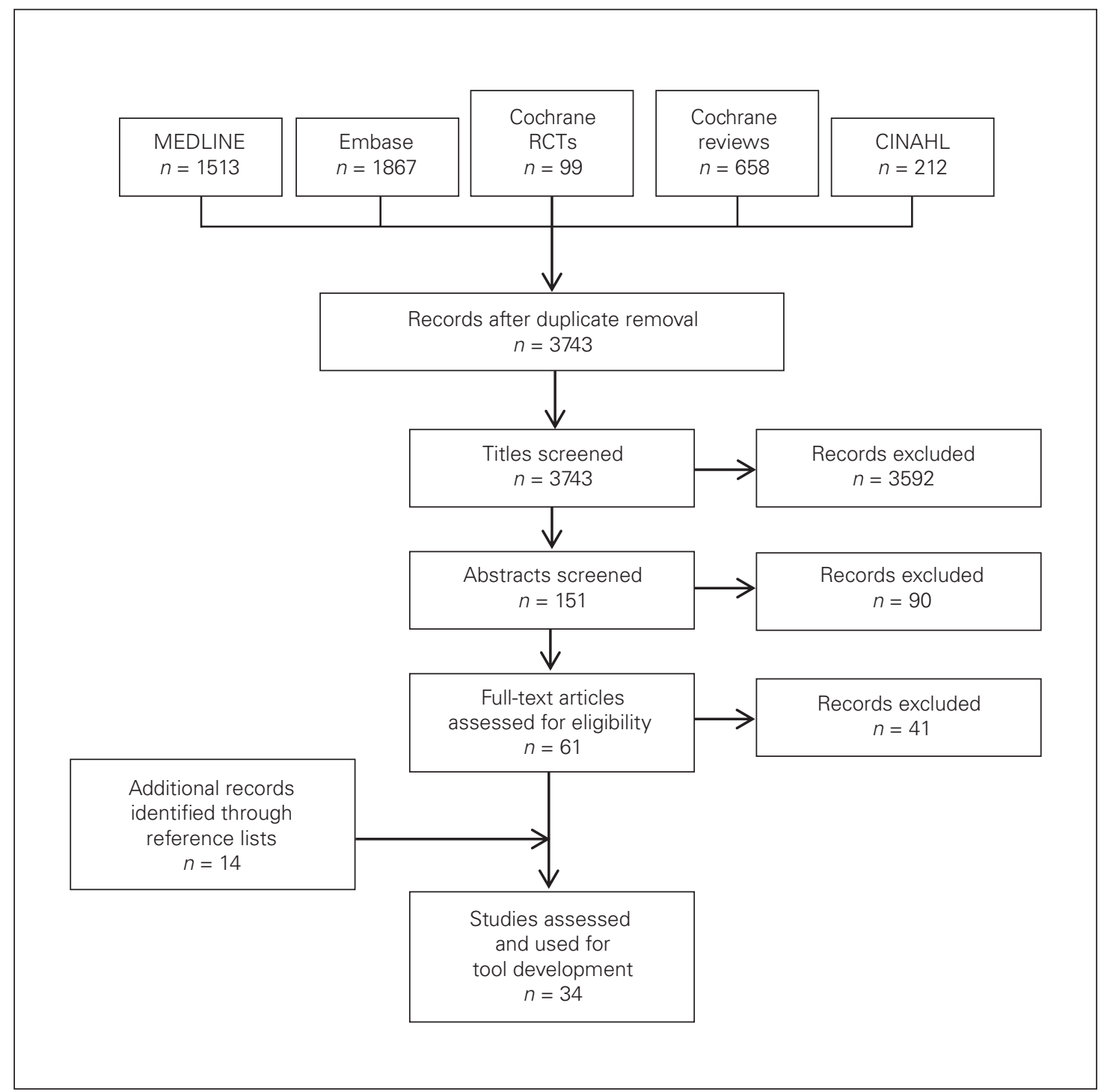

Fig. 1. Flow diagram showing article selection for systematic review. RCT = randomized controlled trial. 
12 weeks. The final version of the wound-assessment tool was refined to 3 major domains that were thought to be the most critical observable factors in monitoring and evaluating wound healing: blistering, peri-incisional skin colour and exudate type (Fig. 2). Each of these domains could be ranked from 1 (best score) to 3 (worst score) on an ordinal scale; the total score a wound could achieve based on this scale ranged from 3 to 9. This would allow for qualitative and quantitative assessment of acute surgical incisions. Based on the clinical piloting in our institutional arthroplasty clinic, these criteria allowed for the creation of a simple multidisciplinary arthroplasty wound-assessment tool, the Simple Multidisciplinary Arthroplasty (SMArt) Wound Tool.

\section{Discussion}

The objective of this study was to systematically develop a wound-assessment tool that could be used to assess the early healing of hip and knee arthroplasty incisions. Our systematic review identified a lack of literature regarding this topic, as well as the need for the development and validation of such a tool. We found no validated acute orthopedic surgical incisional wound-assessment tools in the

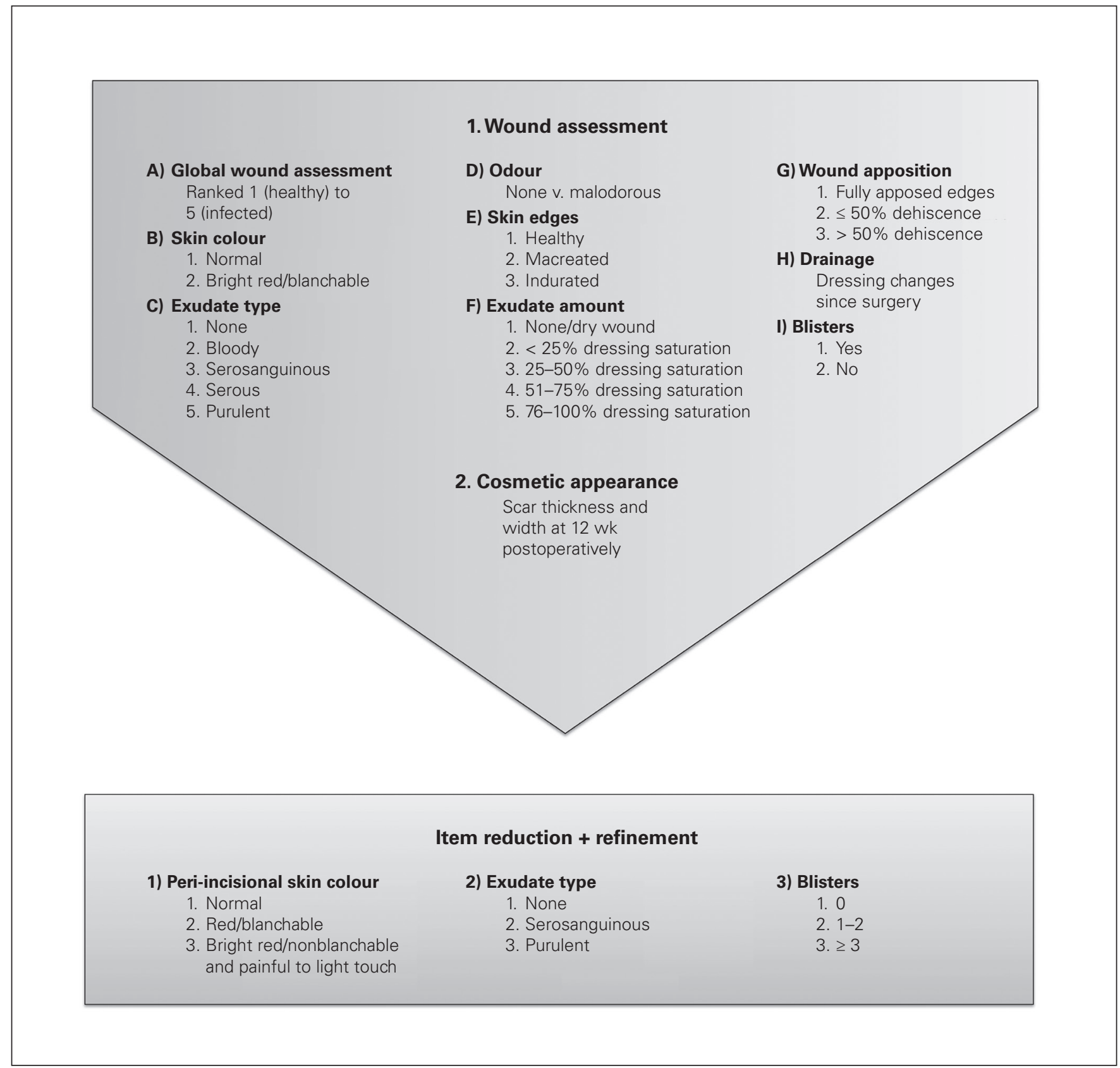

Fig. 2. Creation of Simple Multidisciplinary Arthroplasty (SMArt) Wound Tool domains. 
literature, and the only validated wound-assessment tool that we did find was in the plastic surgery literature regarding the assessment of scar cosmesis 12 weeks postoperatively. ${ }^{9,10}$

Our findings illustrate the paucity of resources and tools for standardizing acute surgical wound assessment. This is particularly concerning considering how much emphasis is put into other aspects of postsurgical care while wound care is largely neglected, despite the ease with which it can be monitored. If we do indeed value holistic medical care, one way in which we could improve communication and documentation among health care professionals is to unify this aspect of surgical care. Some may argue that a standardized method of wound care may increase assessment length and overly complicate this process without added benefit. However, the surgical literature suggests the opposite, indicating that wound assessment is not as simple, innate or universal as some would wish to believe. Proper documentation of wounds is not universally performed, and, when it is done, it is often incomplete and ad hoc. ${ }^{7}$ In addition, doctors and nurses often comment on different aspects of wound care, depending on what they believe to be the most important and relevant. ${ }^{7}$ This disconnect decreases the relevance of medical documentation and reduces multidisciplinary collaboration.

The clinical application of the SMArt Wound Tool to standardize wound assessment across health care professionals has potential benefits. Use of the tool will better assess the progression of wound healing on serial examinations and will aid with the identification of wounds with poor healing potential. The most concerning of postoperative complications is surgical site infection. The SMArt Wound Tool would potentially be most effective in identifying concerning signs of superficial surgical site infection, which include at least 1 of the following within 30 days of surgery: purulent drainage from the surgical site, isolation of organisms from the surgical site, and pain, tenderness, swelling, heat or redness. ${ }^{11,12}$ We believe that our tool is able to effectively capture most of these clinical signs without the addition of nonspecific signs of postsurgical inflammation that could cloud clinical judgment. ${ }^{13-16}$ Superficial surgical site infection increases the risk of deep site infection, and early identification and triage of patients with a superficial infection can dramatically alter a patient's clinical course. ${ }^{15}$ For instance, a patient who has undergone TJA and has been identified as being at high risk for wound complications by means of our tool could potentially receive early intervention. This could range from early administration of antibiotics to more invasive procedures such as irrigation, débridement and polyethylene liner exchange.

The SMArt Wound Tool also has applications in an academic setting as a future research resource. Many of the prospective clinical trials in our systematic review aimed to compare the effect of various wound dressings on wound healing. Without any means of standardization or any justification for the inclusion of various woundassessment domains, the results of these studies are difficult to validate and compare. ${ }^{17}$ A standardized tool has the possible advantage of reducing interobserver reliability, making it optimal for prospective or multicentre studies.

\section{Strengths and limitations}

Strengths of this study include the fact that the development and refinement of the items by an expert panel lend face validity to the SMArt Wound Tool, as compared to the current gold standard of wound assessment, which is single-physician clinical judgment. The diversity of included studies also makes the study results and the wound-assessment tool generalizable to all surgical specialties. This means that the SMArt Wound Tool lends itself to more universal and cross-specialty applications. Furthermore, all of the criteria in our assessment tool can be observed without the need for direct patient contact. This has advantages for remote assessment of wounds using photographs and videos, as these media have already been shown to portray wound appearance accurately. ${ }^{18}$ Finally, we were able to complete the initial objective of our study by performing a sensitive literature search, systematically reviewing the included studies and creating a wound-assessment tool. However, we have yet to validate the tool in clinical practice. Further investigation is required to assess the content and construct validity as well as reliability. This is currently underway in our central intake arthroplasty clinic. A major limitation of this review was the lack of high-quality, relevant studies. We derived many of the wound-assessment domains from narrative reviews or nonvalidated wound-assessment tools. As such, some subjectivity was required when analyzing similar wound terminology or when refining the wound-assessment tool during panel discussions. There was also a lack of orthopedic and arthroplasty literature, which makes the SMArt Wound Tool less specific for patients undergoing TJA.

\section{Conclusion}

There is currently a need for a validated tool to assess the healing of orthopedic surgical incisions, for both clinical and research applications. The SMArt Wound Tool is a systematically developed wound-assessment tool created for assessing acute incisional surgical wounds. It provides a simple, objective method of assessing arthroplasty incisions for the presence of wound complications during the initial healing phase in a multidisciplinary environment. 
Acknowledgements: The authors acknowledge the panel of wound experts as well as Pamela Grossman, Peter Faris and Robert Korley for their guidance in developing the research protocol and arranging for the expert round-table discussions.

Affiliations: From the Section of Orthopaedic Surgery, Department of Surgery, Cumming School of Medicine, University of Calgary, Calgary, Alta. (Kooner, Kendal); the Department of Orthopaedics, Dalhousie University, Saint John, NB (Sheehan); and the Division of Orthopaedic Surgery, McMaster University, Hamilton, Ont. (Johal).

Competing interests: None declared.

Contributors: B. Sheehan and H. Johal designed the study. All authors acquired and analyzed the data, wrote and reviewed the article, and approved the final version for publication.

\section{References}

1. de Fatima de Pina M, Ribeiro AI, Santos C. Epidemiology and variability of orthopaedic procedures worldwide. In: Bentley G, editor. European instructional lectures. European Instructional Lectures vol. 11. Berlin, Heidelberg: Springer; 2011:9-19.

2. Urquhart DM, Hanna FS, Brennan SL, et al. Incidence and risk factors for deep surgical site infection after primary total hip arthroplasty: a systematic review. 7 Arthroplasty 2010;25:1216.

3. Klouche S, Sariali E, Mamoudy P. Total hip arthroplasty revision due to infection: a cost analysis approach. Orthop Traumatol Surg Res 2010;96:124-32.

4. Daines BK, Dennis DA, Amann S. Infection prevention in total knee arthroplasty. 7 Am Acad Orthop Surg 2015;23:356-64.

5. Birchall L, Taylor S. Surgical wound benchmark tool and best practice guidelines. Br 7 Nurs 2003;12:1013-23.

6. Vuolo JC. Assessment and management of surgical wounds in clinical practice. Nurs Stand 2006;20:46-56.

7. Gartlan J, Smith A, Clennett S, et al. An audit of the adequacy of acute wound care documentation of surgical inpatients. 7 Clin Nurs 2010;19:2207-14.
8. Langemo D, Anderson J, Hanson D, et al. Measuring wound length, width, and area: Which technique? Adv Skin Wound Care 2008;21: $42-5$.

9. Duncan JAL, Bond JS, Mason T, et al. Visual analogue scale scoring and ranking: A suitable and sensitive method for assessing scar quality? Plast Reconstr Surg 2006;118:909-18.

10. Bush J, Duncan JAL, Bond JS, et al. Scar-improving efficacy of avotermin administered into the wound margins of skin incisions as evaluated by a randomized, double-blind, placebo-controlled, phase II clinical trial. Plast Reconstr Surg 2010;126:1604-15.

11. Dumville JC, Gray TA, Walter CJ, et al. Dressings for the prevention of surgical site infection. Cochrane Database Syst Rev 2014;(9): CD003091.

12. Cutting KF, White R. Defined and refined: criteria for identifying wound infection revisited. Br 7 Community Nurs 2004;9: S6-15.

13. Miller AG, Swank ML. Dermabond efficacy in total joint arthroplasty wounds. Am 7 Orthop 2010;39:476-8.

14. Vaughan P, Haworth J, Humphrey J, et al. Optimal closure of surgical wounds in forefoot surgery: Are adhesive strips beneficial? Acta Orthop Belg 2006;72:731-3.

15. Abuzakuk TM, Coward P, Shenava Y, et al. The management of wounds following primary lower limb arthroplasty: a prospective, randomised study comparing Hydrofiber and central pad dressings. Int Wound 7 2006;3:133-7.

16. Ravenscroft MJ, Harker J, Buch KA. A prospective, randomised, controlled trial comparing wound dressings used in hip and knee surgery: Aquacel and Tegaderm versus Cutiplast. Ann R Coll Surg Engl 2006;88:18-22.

17. Kottner J, Dassen T, Tannen A. Inter- and intrarater reliability of the Waterlow pressure sore risk scale: a systematic review. Int 7 Nurs Stud 2009;46:369-79.

18. Houghton PE, Kincaid CB, Campbell KE, et al. Photographic assessment of the appearance of chronic pressure and leg ulcers. Ostomy Wound Manage 2000;46:20-6. 